\title{
Electron Microscopy of the Operation of Nanoscale Devices
}

\author{
John Cumings ${ }^{1}$, David Goldhaber-Gordon ${ }^{1}$, A. Zettl ${ }^{2,3}$, M.R. McCartney ${ }^{4}$, and J. C. H. \\ Spence $^{5}$ \\ 1 Department of Physics, Stanford University, Stanford, California; \\ 2 Department of Physics, University of California, Berkeley, California; \\ 3 Materials Sciences Division, Lawrence Berkeley National Lab, Berkeley, California; \\ 4 Center for Solid State Science, Arizona State University, Tempe, Arizona; \\ 5 Department of Physics and Astronomy, Arizona State University, Tempe, Arizona.
}

A transmission electron microscope (TEM) is much more than just a tool for imaging the static state of materials. To demonstrate this, we present work on studying the mechanical and electrical properties of carbon nanotube devices. Multiwall carbon nanotubes are concentrically stacked tubular sheets of graphite, where the spacing between each cylinder is simply the natural spacing of graphite. Using a custom-built in-situ nanomanipulation probe, we have shown that it is possible to slide the nanotube layers in a telescopic extension mode that exhibits low friction, demonstrating the potential of nanotubes as the ultimate synthetic nanobearing.[1] During this telescopic extension, the electrical resistance of the nanotube devices increases, opening the possibility that these devices can also be used as nanoscale rheostats.[2]

We also briefly describe work on using electron holography inside a TEM to study the electric field distribution in nanotube field-emission devices and on using a nanotube itself as a biprism for electron holography (Figure 2). $[3,4]$ These measurements together demonstrate the wealth of information that can be obtained and frontiers that can be opened by putting operational nanodevices inside an electron microscope.

With the power of electron microscopy in imaging nanoscale devices at high speeds, the future for studying these systems is bright. We are presently developing techniques to fabricate nanoscale electronic devices on the surface of commercially available silicon nitride membranes (SPI, inc.). This will allow the investigation of a large variety of tailor-assembled nanoscale systems to be studied during operation. We are also expanding the analytical capabilities available inside a TEM to include thermal imaging. This will allow the investigation of the local generation and dissipation of heat during device operation.

\section{References:}

[1] J. Cumings, P. G. Collins, and A. Zettl, Nature 406, 586 (2000)

[2] J. Cumings and A. Zettl, Phys. Rev. Lett. 93, 086801 (2004)

[3] J. Cumings, A. Zettl, M. R. McCartney, and J. C. H. Spence, Phys. Rev. Lett. 88, 056804 (2002)

[4] J. Cumings, A. Zettl, and M. R. McCartney, Microsc. Microanal. 10, 420 (2004) 

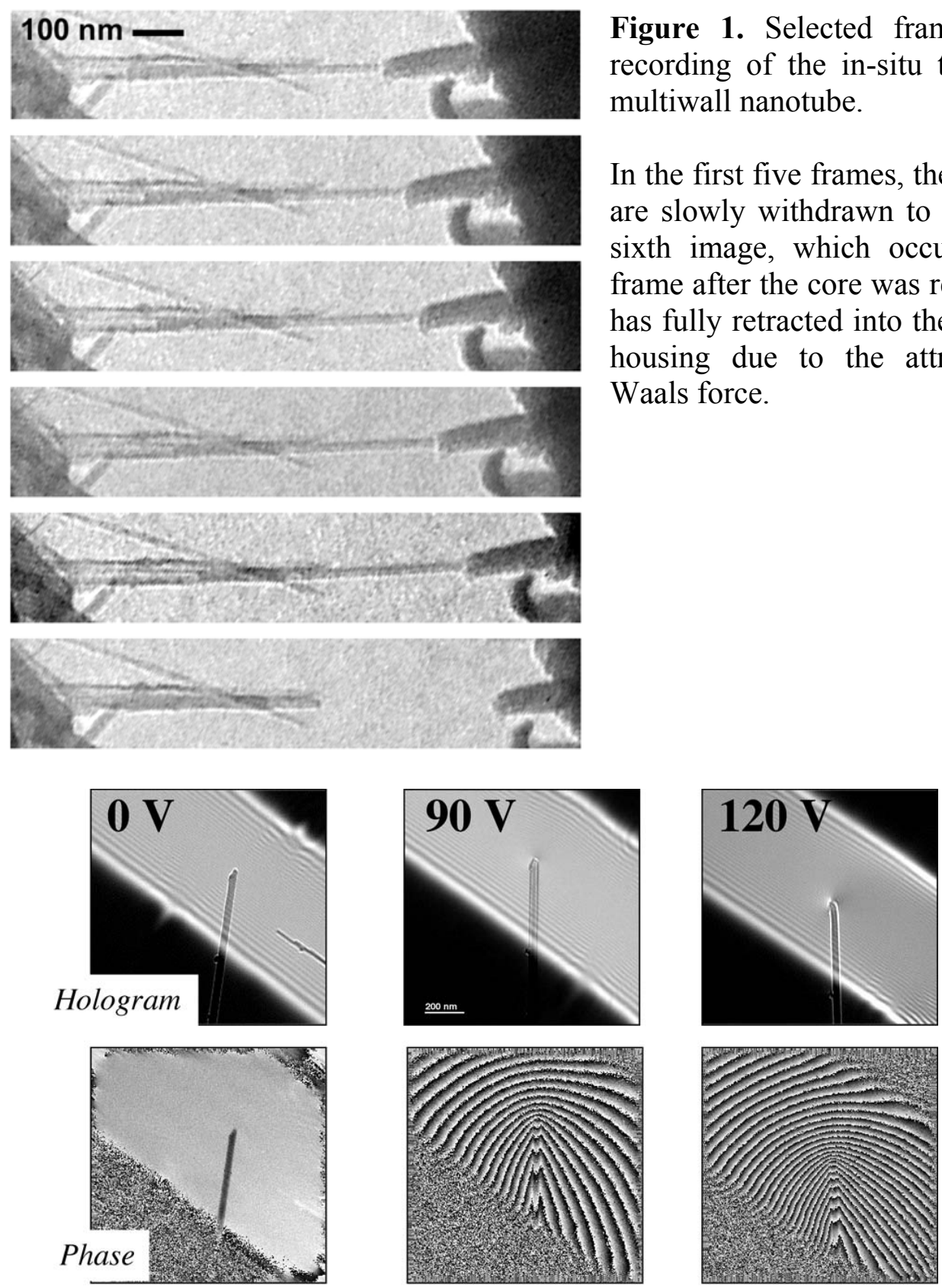

In the first five frames, the core nanotubes are slowly withdrawn to the right. In the sixth image, which occurred one video frame after the core was released, the core has fully retracted into the outer nanotube housing due to the attractive van der Waals force.

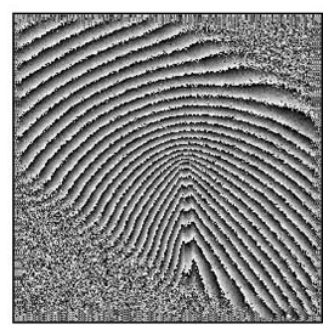

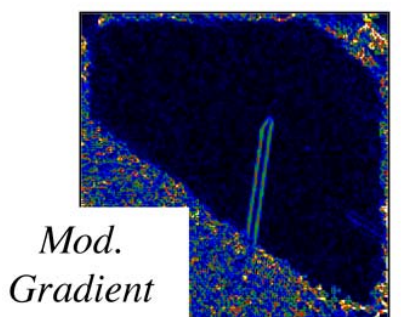

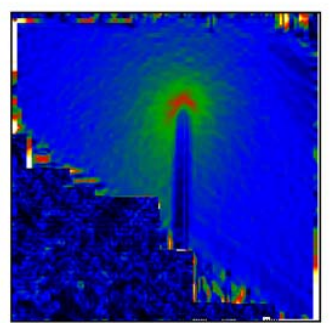

Figure 2 Phase shift and phase gradient maps extracted from holograms of a fieldemitting nanotube. 